Article

\title{
A Rigid Cuckoo Search Algorithm for Solving Short-Term Hydrothermal Scheduling Problem
}

\author{
Cui Zheyuan ${ }^{1,2,+}$, Ali Thaeer Hammid ${ }^{3,+}$, Ali Noori Kareem ${ }^{4}$, Mingxin Jiang ${ }^{5} \mathbb{D}$, Muamer N. Mohammed ${ }^{6}$ \\ and Nallapaneni Manoj Kumar ${ }^{7, *(D)}$
}

1 School of Computer Science, Baoji University of Arts and Sciences, Baoji 721007, China; syxz234@gmail.com 2 Faculty of Management and Economics, Universiti Pendidikan Sultan Idris, Tanjong Malim 35900, Malaysia

3 Computer Engineering Techniques Department, Faculty of Information Technology,

Imam Ja'afar Al-Sadiq University, Baghdad 10012, Iraq; a.t.hammid@sadiq.edu.iq

4 Computer Engineering Department, Bilad Alrafidain University College, Ba'aqubah, Diyala 32001, Iraq; dr.alinoori@bauc14.edu.iq

5 Faculty of Electronic Information Engineering, Huaiyin Institute of Technology, Huaian 223003, China; jmx@hyit.edu.cn

6 Information Technology Department, Community College of Qatar (CCQ), Doha 00974, Qatar; muamer.almafrachi@ccq.edu.qa

7 School of Energy and Environment, City University of Hong Kong, Kowloon, Hong Kong, China

* Correspondence: mnallapan2-c@my.cityu.edu.hk

+ Both have contributed equally (first and co-first author).

Citation: Zheyuan, C.; Hammid, A.T.; Kareem, A.N.; Jiang, M.; Mohammed, M.N.; Kumar, N.M. A Rigid Cuckoo Search Algorithm for Solving Short-Term Hydrothermal Scheduling Problem. Sustainability 2021, 13, 4277. https://doi.org/ $10.3390 /$ su13084277

Academic Editor: Md Rabiul Islam

Received: 27 February 2021

Accepted: 10 April 2021

Published: 12 April 2021

Publisher's Note: MDPI stays neutral with regard to jurisdictional claims in published maps and institutional affiliations.

Copyright: (c) 2021 by the authors. Licensee MDPI, Basel, Switzerland. This article is an open access article distributed under the terms and conditions of the Creative Commons Attribution (CC BY) license (https:// creativecommons.org/licenses/by/ $4.0 /)$.

\begin{abstract}
The key criteria of the short-term hydrothermal scheduling (StHS) problem is to minimize the gross fuel cost for electricity production by scheduling the hydrothermal power generators considering the constraints related to power balance; the gross release of water, and storage limitations of the reservoir, and the operating limitations of the thermal generators and hydropower plants. For addressing the same problem, numerous algorithms were being used, and related studies exist in the literature; however, they possess limitations concerning the solution state and the number of iterations it takes to reach the solution state. Hence, this article proposes using an enhanced cuckoo search algorithm (CSA) called the rigid cuckoo search algorithm (RCSA), a modified version of the traditional CSA for solving the StHS problem. The proposed RCSA improves the solution state and decreases the iteration numbers related to the CSA with a modified Lévy flight. Here, the movement distances are divided into multiple possible steps, which has infinite diversity. The effectiveness of RCSA has been validated by considering the hydrothermal power system. The observed results reveal the superior performance of RCSA among all other compared algorithms that recently have been used for the StHS problem. It is also observed that the RCSA approach has achieved minimum gross costs than other techniques. Thus, the proposed RCSA proves to be a highly effective and convenient approach for addressing the StHS problems
\end{abstract}

Keywords: rigid cuckoo search algorithm; hydropower plants; minimizing fuel cost; short-term hydrothermal scheduling; Lévy flights

\section{Introduction}

The short-term hydrothermal scheduling (StHS) aims to minimize thermal units gross fuel cost by optimally scheduling the thermal generators and hydropower units' operation for a given time. The support of numerous algorithms does the optimal scheduling, and there exist many studies in the literature. Owing to the StHS objective function's nonlinear nature, Lagrange multipliers and gradient techniques must be employed. On the other side, considering the linearized behavior produces non-optimal solutions that appear in immense revenue generation losses, which was also done through scheduled operations [1,2]. In the StHS, the electrical load sharing requests have been optimally specified between thermal generators and hydropower units by appropriately planning 
the hydrothermal system's operation, meeting the StHS key criteria, i.e., minimum fuel cost [3].

As mentioned earlier, many studies used optimization techniques to address the StHS problem. In ref. [3], a detailed summary of those studies along with a brief discussion on various optimization techniques and algorithms can be found. Apart from those, there exist few recent studies on the StHS problem that used a genetic algorithm (GA) [4], enhanced GA (EGA), particle swarm optimization (PSO), and enhanced PSO (EPSO) [5], classical evolutionary programming (CEP), fast evolutionary programming (FEP), and improved FEP (IFEP) [6], grasshopper optimization algorithm (GOA) [7], adaptive particle swarm optimization (APSO), modified APSO (MAPSO) [7], modified differential evolution (MDE) [8], improved PSO (IPSO) [9], teaching learning-based optimization (TLBO) [10], one rank cuckoo search algorithm (ORCSA) [11], running IFEP (RIFEP) [12], gradient search techniques (GS) [13], simulated annealing approach (SA) [14], clonal selection roy (CSR) [15], krill herd algorithm (KHA) [16], and sequential quadratic programming (SQP) [17].

All the above-suggested algorithms have contributed significantly in addressing the StHS problem; however, they possess limitations concerning the solution state and the number of iteration it takes to reach the solution state. Lately, the cuckoo search algorithm (CSA) has been proposed for economic dispatch problems; however, Yang and Deb, in 2009, used the CSA for the first time to solve optimization problems [18]. CSA is one of the metaheuristic algorithms supporting several rule parameters. It mimics the parasitism of several cuckoo kinds by depositing their eggs in the host bird nests for other kinds. Upon seeing the advantage of CSA in solving the optimization problem-solving ability, it was later used to solve non-convex and economic dispatch problems [19]. More recently, the CSA was even used for addressing the StHS problem; see in ref [20] for details. The results of ref. [20], suggested that CSA is a practical approach with improved performance, which is better than all other cases containing high-rate nonlinearity like valve point loads. However, the traditional CSA algorithm suffers from a limitation of step-length variation, which is crucial in attaining the solution. Hence, this article proposes using an enhanced CSA called rigid cuckoo search algorithm (RCSA), a modified version of the traditional CSA for solving the StHS problem.

In the proposed RCSA technique, we consider a modified Lévy flight in which the step-lengths have been classified based on its new specific possibility configuration, which produces unlimited variation. The sequential jumps or steps of a cuckoo necessarily create a stochastic step method that adopts a power law for step length configuration resulting in large final step-sizes. Hence, it is wise to make a stochastic step in a biased process with many stochastic step sizes. As a result, RCSA for the StHS optimization problems enhances the optimization convergence accuracy and rate. Additionally, the effectiveness of RCSA has been examined and validated on complicated optimization problems of hydrothermal scheduling.

The article is structured in three sections, where Section 2 provided the detailed mathematical modeling of the hydrothermal power system and the RCSA implementation. In Section 3, the results are discussed and compared with other algorithms. Finally, the concluding remarks were provided in Section 4.

\section{Methods}

\subsection{Mathematical Model of the Hydrothermal System}

This section provides a mathematical model of the hydrothermal power system that we used for optimization. Between the two generators (hydro and thermal), the input fuel cost is negligible in hydropower generating units due to water availability as a free source. However, it is different from thermal power generators; hence, our main aim was to minimize the gross input fuel cost of thermal power generating units while producing the energy by using hydro resources significantly. Considering the above condition, we 
selected the objective function that is shown in Equation (1). Additionally, the considered constraints for solving the StHS problem are provided in Equations (2) to (9) [21-23].

Objective function:

$$
\min F=\sum_{j=1}^{J} f_{i}\left(P_{T j}\right)
$$

Constraints:

The constraint related to the balance of power generation-load is given by:

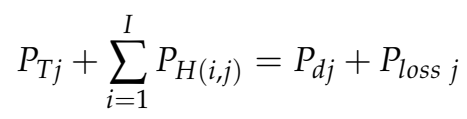

The hydropower generation $\left(P_{H(i, j)}\right)$ is a rate function of water release and is given by:

$$
P_{H(i, j)}=\phi(q)
$$

The water volume stored in the reservoir can be given by:

$$
X_{i(j+1)}=X_{i j}-q_{i(j+1)}-s_{i(j+1)}+r_{i(j+1)}
$$

Operational periods of thermal power generators have been restrained according to their capacities are given by:

$$
P_{T, \min } \leq P_{T} \leq P_{T, \max }
$$

Operational periods of hydropower generators have been restrained according to their capacities are given by:

$$
P_{H, \min } \leq P_{H} \leq P_{H, \max }
$$

The constraints related to water release rate limitations are represented by:

$$
q_{i, \min } \leq q_{i, j} \leq q_{i, \max }
$$

The constraints related to initial and final reservoir water volume are given by

$$
X_{i}^{0}=X_{i}(0), X_{j}^{0}=X_{j}(0)
$$

The constraints related to reservoir limitations of water storage are given by

$$
X_{i, \min } \leq X_{i, j} \leq X_{i, \max }
$$

\subsection{Cuckoo Search Algorithm and Lévy Flights}

The CSA is a metaheuristic search algorithm developed by Yang and Deb [18]. Compared to other algorithms, the CSA is a unique technique based on a heuristic evolutionary algorithm's population to solve optimization problems like the StHS problem with easy execution and few adjustable parameters. The CSA algorithm rule is related to the compulsory brood parasitic performance and Lévy flight performance of several birds and fruit flies. Below are three essential concepts that are helpful throughout the rule exploration.

- Concept 1 . Individually all cuckoos produce one single egg at a time that regards a proposed solution and randomly throws its egg up into the wanted nest among the set number of possible host nests.

- Concept 2. The egg of high quality thrown up in the best nests regards a better solution transferred to the subsequent generation.

- Concept 3. The possible number of host nests is constant, and the probability that the host bird can find a nest is indicated by the probability constant, $\mathrm{Pa}$, with range $[0,1]$. Hence, it may either discard the egg or leave this nest and then build a new nest entirely in a different place. 
Based on the above-listed principles in the order of steps, cuckoos naturally explore food options in a random or semi-random way. A cuckoo forgoing route is adequately an unexpected step due to the subsequent progress based on both the present position and the passage possibility to the next position. These unexpected steps can be modeled mathematically, and in the literature, it was explained that the flight performance could illustrate the ideal aspects by the Lévy flights concept [24]. A Lévy flight is a random step in which every step's length is classified based on a heavy-tailed possibility configuration. Afterward, the length from the start point of the random steps leads to a steady configuration in some steps.

\subsection{Rigid Cuckoo Search Algorithm}

According to the three rules mentioned previously in Section 2.2, for finding the generation of new solutions, i.e., $S_{i}(t+1)$ for the $i^{\text {th }}$ cuckoo, the Lévy flight shown in Equation (10) is achieved.

$$
S_{i}(t+1)=S_{i}(t)+\alpha \oplus L e^{\prime} v y(\lambda)
$$

where $\alpha$ must be more than zero 0 (for all of the step size), according to the range of the interest problem.

The product $\oplus$ indicates elementwise multiplication. This study considers Lévy flight in which the step-lengths have been distributed due to the subsequent possibility allocation, which has an unlimited variation, see in Equation (11)

$$
L e^{\prime} \text { vy } u=t^{-\lambda}, 0.999<\lambda \leq 3.079
$$

Here, the steps made by any single cuckoos are typically represented in a stochastic manner that follows a power-law of allocation for the step-length with a thick tailpiece. It is worth noting that, if egg cuckoos were quite similar to the host eggs, then these egg cuckoos' have few possibilities, which can be identified. Therefore, the fitness must relate to the variance solutions. Hence, the RCSA does entire stochastic steps in a biased approach with several unplanned step dimensions for obtaining the solutions. There are three parameters $P a, \lambda$, and $\alpha$, which are included in the CSA to support this technique to obtain universally and regionally enhanced solutions. The parameters $P a$ and $\alpha$ are quite significant in fine-tuning vectors of the solution and can be employed to set the algorithm convergence rate. The conventional CSA algorithm uses established values for both $\mathrm{Pa}$ and $\alpha$, defined in the initialization stage and cannot be replaced later. Additionally, the iterations number to obtain an optimal solution is another disadvantage of conventional CSA. If there were the low-value of $P a$ and the high-value of $\alpha$, this technique's execution would be weak and manages to a significant and result in the iterations number increment. Moreover, if there were the high-value of $P a$ and the low-value of $\alpha$, the convergence speed would be immense, but it probably cannot obtain the best solutions. Hence, we used the RCSA approach to solve the StHS problem; the flow chart presented in Figure 1 illustrated solving methodology. 


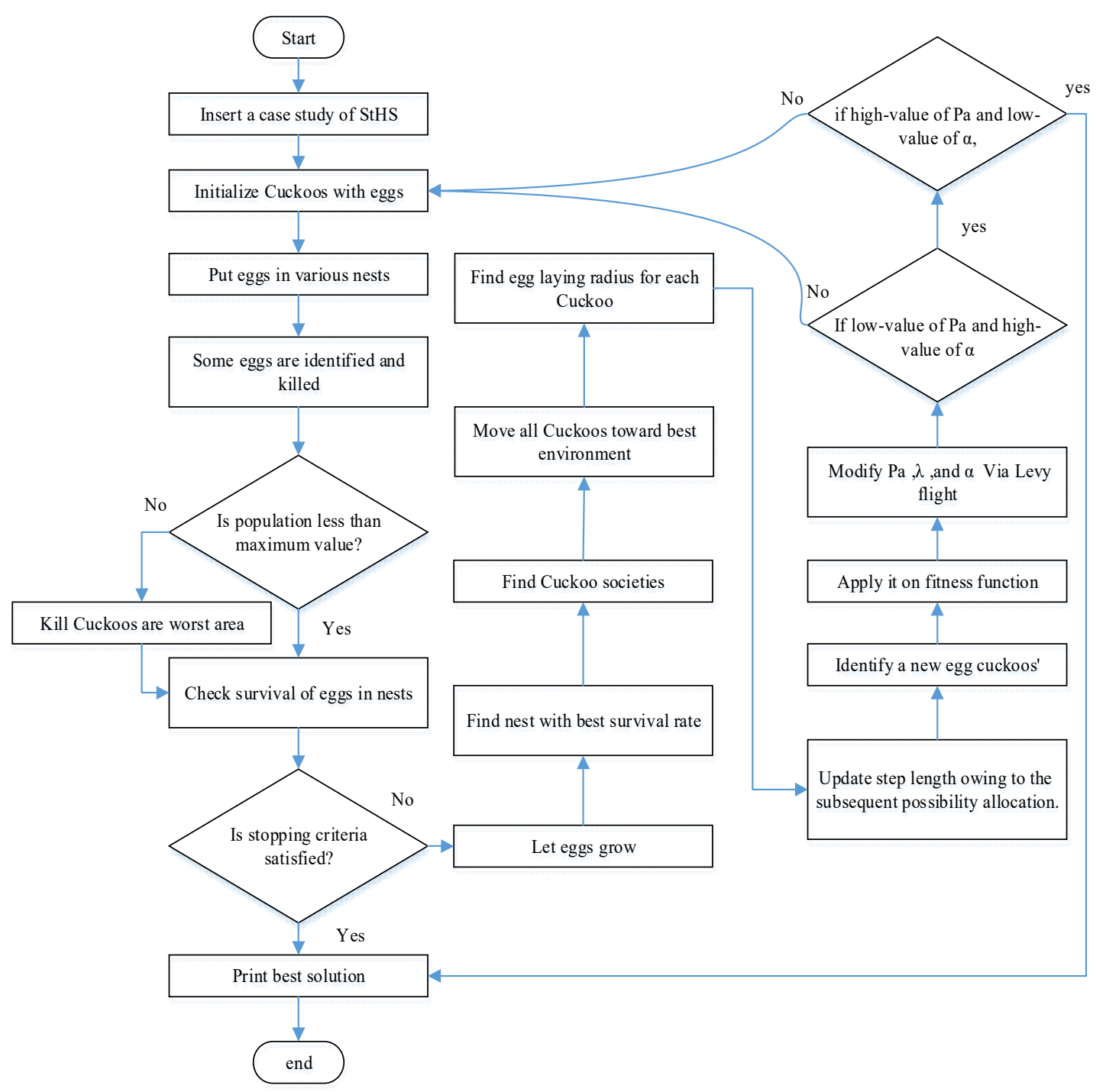

Figure 1. Flow chart showing the steps used in the rigid cuckoo search algorithm (RCSA) approach for solving the short-term hydrothermal scheduling (StHS) problem.

The essential disparity between the improved CSA (i.e., RCSA) and CSA is modifying $P a$ and $\alpha$. To enhance the CSA technique's achievement and minimize the disadvantages that lie on established values of $P a$ and $\alpha$, the improved CSA technique employs variable values for $P a$ and $\alpha$. Hence, in the new steps, the values of $P a$ and $\alpha$ obligate to be large and execute the technique to improve the solution vectors. Nevertheless, these values must be reduced in definitive productions to succeed in a more regular fine-tuning of solution vectors. The values of $\mathrm{Pa}$ and $\alpha$ are physically replaced with the production number, see in Equations (12)-(14)

$$
\begin{gathered}
P_{a}\left(I_{T}\right)=P_{a, \text { max }}-\frac{P_{a, \text { max }}-P_{a, \text { min }}}{I_{\mathcal{C}}} \times I_{T} \\
\alpha\left(I_{T}\right)=\alpha_{\max } \cdot e^{\left(c . I_{T}\right)} \\
c=\frac{\operatorname{Ln}\left(\frac{\alpha_{\max }}{\alpha_{\min }}\right)}{I_{c}}
\end{gathered}
$$

However, it becomes difficult to find the new solutions with the restrained optimization problem. Hence, there is a need for converting the restrained optimization problem to unrestrained and is as follows: 
The optimization problem is expressed as follows in Equation (15)

$$
\operatorname{Min} f_{i}\left(P_{T j}\right) \text {, s.t. }: R_{j} \leq 0 j=1,2, \ldots, N_{P}
$$

So, in such cases, the impossible solution must be adjusted to be possible solutions, which prevents the severity of restrictions. For this matter, a discipline function has been used to change the restrained optimization problem in Equation (16) to the unrestrained one in Equation (17)

$$
\operatorname{Max} F^{*}=f_{i}\left(P_{T j}\right)-\lambda \sum_{i=1}^{N_{P}} \min \left(0, R_{j}\right)
$$

Based on the discipline coefficient, the maximization problem of Max $F^{*}$ is made equal to Min $F^{*}$. It is represented as follows, see in Equation (17).

$$
\operatorname{Min} F^{*}=-f_{i}\left(P_{T j}\right)+\lambda \sum_{i=1}^{N_{P}} \min \left(0, R_{j}\right)
$$

\subsection{Implementation of RCSA on a Hydrothermal System}

Step-1. In the StHS problem, the influential variables such as the release rate of water for the whole plants for several hours and thermal unit production for the entire period are chosen irregularly within the operating limitations. The storage capacity of every reservoir has been estimated using Equation (4), the generation of hydro plants has been calculated using Equation (3). Subsequently, the thermal power generation has been computed by applying Equation (2). The population of the host nest $\left(N_{E}\right)$ has been explained as:

$$
Y=\left[Y_{1}, Y_{2}, \ldots, Y_{N_{E}}\right]^{L}
$$

where every nest $Y_{i}$ is expressed as:

$$
Y=\left[\begin{array}{cccccccccc}
q_{1,1}^{i} & \cdots & q_{1, j}^{i} & \cdots & q_{1, N h}^{i} & P_{T_{1,1}}^{i} & \cdots & P_{T_{1, j}}^{i} & \cdots & P_{T_{1, N h}}^{i} \\
\cdots & \cdots & \cdots & \cdots & \cdots & \cdots & \cdots & \cdots & \cdots & \cdots \\
q_{k, 1}^{i} & \cdots & q_{k, j}^{i} & \cdots & q_{k, N h}^{i} & P_{T_{k, 1}}^{i} & \cdots & P_{T_{k, j}}^{i} & \cdots & P_{T_{1, N h}}^{i} \\
\cdots & \cdots & \cdots & \cdots & \cdots & \cdots & \cdots & \cdots & \cdots & \cdots \\
q_{N h, 1}^{i} & \cdots & q_{N h, j}^{i} & \cdots & q_{N h, n h}^{i} & P_{T_{N h, 1}}^{i} & \cdots & P_{T_{N h, j}}^{i} & \cdots & P_{T_{N h, n h}}^{i}
\end{array}\right]
$$

Step-2. Set the production number.

Step-3. Compute the objective function using Equation (17). With the equation of restraints, many restrictions irregularly have been limited. Then, enhanced fuel cost has been calculated as Equation (19).

$$
F^{* *}=F^{*}+\sum_{k=1}^{N_{C}}\left(\lambda_{k} \times V i o_{k}^{2}\right)
$$

Step-4. The modern solution has been created by using Levy flights. The new solution's computation has been built in the preceding best nest by using Levy flights. For this technique, the optimal way for levy flights has been computed by Yang XS's contribution, Deb S [18]. The new solution has been presented in Equation (20)

$$
Y_{i, \text { new }}=Y_{i, \text { best }}+\left(\alpha \times \text { rand }_{2} \times \Delta Y_{i, \text { new }}\right)
$$


where $\alpha>0$ is the updated step size, rand $_{2}$ is a typical number of allocated stochastic and $\Delta Y_{i, \text { new }}$ has been computed, see Equations (21) and (22):

$$
\begin{gathered}
\Delta Y_{i, \text { new }}=\varepsilon \times \frac{\rho_{1}(\psi)}{\rho_{2}(\psi)} \times\left(Y_{i, \text { best }}-G_{\text {best }}\right) \\
\varepsilon=\frac{\operatorname{rand}_{A}}{\left|\operatorname{rand}_{B}\right|^{\frac{1}{\psi}}}
\end{gathered}
$$

where $\operatorname{rand}_{A}$ and $\operatorname{rand}_{B}$ are a couple of commonly allocated stochastic variables with a standard deviation $\rho_{1}(\psi)$ and $\rho_{2}(\psi)$ that has been determined by Equations (23) and (24), respectively.

$$
\begin{gathered}
\rho_{1}(\psi)=\left[\frac{\Phi(1+\psi) \times \sin \left(\frac{\pi \psi}{2}\right)}{\Phi\left(\frac{1+\psi}{2}\right) \times \psi \times 2^{\left(\frac{\psi-1}{2}\right)}}\right]^{\frac{1}{\psi}} \\
\rho_{2}(\psi)=1
\end{gathered}
$$

where $\psi$ is between the range from 0.29 to 2.01 and the obtained new solution must satisfy all associated constraints for both of $\psi$ and $\Phi$ as well.

Step-5. The effect of the detection of an alien egg in a nest of a host bird with Pa's possibility produces a new solution for the problem comparable with the Levy flights. The new solution has been computed as following Equations (25)-(27):

$$
\Delta Y_{i, d i s}=Y_{i, b e s t}+\left(k \times \Delta Y_{i, d i s}\right)
$$

where $k$ was the modernized coefficient defined built in the possibility of a host bird to find out an alien egg in its nest:

$$
k=\left\{\begin{array}{cc}
1 & \text { if rand } \\
0 & >P_{a} \\
\text { otherwise }
\end{array}\right.
$$

The increment value of $\Delta Y_{i, \text { dis }}$ has been defined by

$$
\Delta Y_{i, \text { dis }}=\operatorname{rand}_{3} \times\left[\operatorname{rand}_{p_{1}}\left(Y_{d, \text { best }}\right)-\operatorname{rand}_{p_{2}}\left(Y_{d, \text { best }}\right)\right]
$$

where $\mathrm{rand}_{3}$ is the allocated random number within [0,1], $\operatorname{rand}_{p_{1}}\left(Y_{d, \text { best }}\right)$ and $\operatorname{rand}_{p_{2}}\left(Y_{d, \text { best }}\right)$ are the random disorder for locations of nests in $\left(Y_{d, \text { best }}\right)$. Once more, for the recently produced solution, its lower and upper limits should please the unit's limitation. The best value has been modernized for every nest $\left(Y_{d, \text { best }}\right)$ and the nest identical to the best fitness function has been defined by Gbest.

Step-6. The technique ends if modern production gives the maximum production number.

\section{Results and Discussion}

This section provides the results of an RCSA application in addressing the StHS problem; hence, a test system of hydrothermal power generating units was considered and is based on the refs $[7,10]$. It involves a complex of four hydro plants and some thermal units regarded as a single equivalent thermal plant. To understand the RCSA technique's feasibility for a more extensive hydrothermal power system, it has been applied on a second test system that involved a cascade of three thermal units and four hydro plants. The real data of this system was collected based on refs [10,25]. The schedule period of $24 \mathrm{~h}$, and every interval equal to $1 \mathrm{~h}$ time, has been considered. The simulation was carried out using MATLAB 9.8, and the computer used is a core i7-8th Gen processor with $2.00 \mathrm{GHz}$ and 8.00 GB RAM. 


\subsection{Parameter Selection}

In the RCSA, there were just five parameters that can be harmonized, involving three major items from the authentic CSA and a couple of other adjustments. Firstly, a couple of parameters that involved the three major items, which influence every recent solution produced from the exploration and exploitation are considered. These aspects are the nests' number $\left(\mathrm{N}_{\mathrm{E}}\right)$ and the possibility of an alien egg to be found out, Pa. In contrast, the number of maximum iterations should have undeviatingly an effect on the optimal solution. Secondly, a couple of other parameters that influence joining the exploration aspect and exploitation aspects are considered. These should be satisfied with the upper and lower limitations and can be adjusted via the Lévy flights power. The obliged with the best solution provides the RCSA technique to enhance its performance and convergence speed. On the other side, the three major parameters from the authentic CSA technique, a couple of others in the justification, were effortless to be selected because they had been clarified by the previously limit equations. After several number of runs with various values of RCSA control parameter, the key control parameter chosen are population $(\mathrm{Np})=100$, maximum iteration $=500$ and value of probability $(\mathrm{Pa})=0.7$.

\subsection{Obtained Results}

The proposed RCSA was executed more than ten confident times within limit range values of Pa from 0.1 up to 0.9 , and a specific version of RCSA was achieved more than 100 confident times. In contrast, the nests' number and maximum iterations number are previously groups to particular values of 10 and 300, respectively. The results contain minimal total cost, average gross cost, maximum gross cost, average calculation time, and standard deviation collected by RCSA, respectively illustrated in Tables 1 and 2.

Table 1. Brief result from proposed RCSA with various values of Pa.

\begin{tabular}{cccccc}
\hline $\mathbf{P}_{\mathbf{a}}$ & Min Cost (\$) & Avg. Cost (\$) & Max Cost (\$) & Std. dev. (\$) & CPU (s) \\
\hline 0.1 & $709,932.115$ & $709,995.586$ & $710,655.745$ & 327.189 & 17.9 \\
0.2 & $709,922.44$ & $710,768.874$ & $711,384.563$ & 599.382 & 17.9 \\
0.3 & $709,911.445$ & $709,994.412$ & $710,989.321$ & 489.733 & $\mathbf{1 7 . 8}$ \\
0.4 & $709,866.727$ & $\mathbf{7 0 9 , 7 4 5 . 2 3 6}$ & $709,999.741$ & 103.937 & 18.1 \\
0.5 & $709,886.651$ & $709,887.698$ & $\mathbf{7 0 9 , 9 8 8 . 2 5 8}$ & 47.653 & 18.6 \\
0.6 & $709,862.129$ & $709,899.987$ & $709,991.951$ & $\mathbf{5 4 . 5 1 2}$ & 18.5 \\
0.7 & $\mathbf{7 0 9 , 8 6 2 . 0 2 7}$ & $709,878.852$ & $709,996.159$ & 59.661 & 18.2 \\
0.8 & $709,901.478$ & $709,910.357$ & $710,920.357$ & 478.225 & 18.3 \\
0.9 & $709,902.685$ & $709,901.753$ & $710,901.753$ & 471.185 & 18.7 \\
\hline
\end{tabular}

Table 2. The optimal solutions achieved by the proposed RCSA technique.

\begin{tabular}{cccccc}
\hline $\mathbf{m}$ & $\mathbf{P}_{\mathbf{D m}}$ & $\begin{array}{c}\mathbf{V}_{\mathbf{m}} \\
\text { (acre-ft) }\end{array}$ & $\begin{array}{c}\mathbf{q}_{\mathbf{m}} \\
\text { (acre-ft/hr) }\end{array}$ & $\begin{array}{c}\mathbf{P}_{\mathbf{s m}} \\
\mathbf{( M W )}\end{array}$ & $\begin{array}{c}\mathbf{P}_{\mathbf{h m}} \\
\mathbf{( M W )}\end{array}$ \\
\hline 1 & 1199 & 101,897 & 1832 & 892 & 300 \\
2 & 1497 & 85,959 & 3328 & 892 & 600 \\
3 & 1098 & 93,847 & 1340 & 892 & 200 \\
4 & 1795 & 59,998 & 4817 & 892 & 900 \\
5 & 948 & 70,428 & 1124 & 783 & 158 \\
6 & 1289 & 59,998 & 2863 & 783 & 509 \\
\hline
\end{tabular}

Based on the solutions present in Tables 1 and 2, the CSA obtained optimal solutions at $\mathrm{Pa}$ from 0.1 up to 0.9 , and RCSA obtained optimal solutions at Pa precisely equal to 0.7. Moreover, RCSA could get less maximum gross cost, less average gross cost, and less standard deviation.

The specific optimum points of the solution involving water release and thermal power production have been illustrated in Table 3 . Thus, it is proved that the proposed RCSA technique effectively attains the solution for the StHS problem with cascaded hydropower 
plants. The load demand and the thermal and hydropower plants' power for every interval of time throughout the scheduling horizon correlating to the best solution for test system 1 is illustrated in Figure 2. For the same aspect, Figure 3 displays the reservoir storage volumes of entire hydro plants and the cost convergence characteristic of the suggested technique.

Table 3. Optimal solution obtained by RCSA for test system 1 .

\begin{tabular}{|c|c|c|c|c|c|}
\hline \multirow{2}{*}{ Hours } & \multicolumn{4}{|c|}{ Water Discharge $\left(\times 10^{4} \mathrm{~m}^{3}\right)$} & \multirow{2}{*}{ Ps (MW) } \\
\hline & Plant 1 & Plant 2 & Plant 3 & Plant 4 & \\
\hline 1 & 9.4884 & 6.1377 & 26.3477 & 13.1009 & 1036.249 \\
\hline 2 & 9.4025 & 6.3104 & 25.2088 & 13.1024 & 1066.243 \\
\hline 3 & 9.197 & 6.256 & 24.504 & 13.1035 & 1049.837 \\
\hline 4 & 8.8096 & 6.3038 & 23.8832 & 13.1003 & 996.409 \\
\hline 5 & 8.5269 & 6.2552 & 22.7287 & 13.1009 & 975.264 \\
\hline 6 & 8.3314 & 6.4278 & 21.9238 & 13.1007 & 1076.828 \\
\hline 7 & 8.4101 & 6.821 & 206226 & 13.1005 & 1294.982 \\
\hline 8 & 8.6081 & 7.2309 & 19.2428 & 13.1023 & 1623.787 \\
\hline 9 & 8.8072 & 7.3387 & 18.5775 & 13.1077 & 1849.655 \\
\hline 10 & 8.465 & 7.5783 & 17.8006 & 13.1163 & 1917.3778 \\
\hline 11 & 8.3429 & 7.7186 & 17.0931 & 13 & 1816.169 \\
\hline 12 & 8.4121 & 7.8032 & 16.9969 & 13.4302 & 1885.493 \\
\hline 13 & 8.2444 & 7.8624 & 16.3144 & 14.6742 & 1786.317 \\
\hline 14 & 8.1224 & 8.0001 & 15.439 & 15.9371 & 1737.617 \\
\hline 15 & 7.9417 & 8.1501 & 14.3596 & 17.2956 & 1652.815 \\
\hline 16 & 7.8102 & 8.5518 & 12.9066 & 18.4244 & 1580.968 \\
\hline 17 & 7.8153 & 8.9344 & 11.4353 & 20.0065 & 1630.883 \\
\hline 18 & 7.6626 & 9.3193 & 10.1008 & 21.4157 & 1639.432 \\
\hline 19 & 7.672 & 9.9655 & 10.1316 & 23.0205 & 1739.29 \\
\hline 20 & 7.5945 & 10.5252 & 10.1043 & 24.2843 & 1787.795 \\
\hline 21 & 7.3908 & 11.1875 & 12.1618 & 25.0985 & 1761.32 \\
\hline 22 & 7.586 & 12.2327 & 12.747 & 25.0636 & 1659.735 \\
\hline 23 & 7.5513 & 13.1386 & 13.2403 & 25.0994 & 1411.135 \\
\hline 24 & 7.4103 & 14.2533 & 13.6662 & 25.0972 & 1177.636 \\
\hline
\end{tabular}

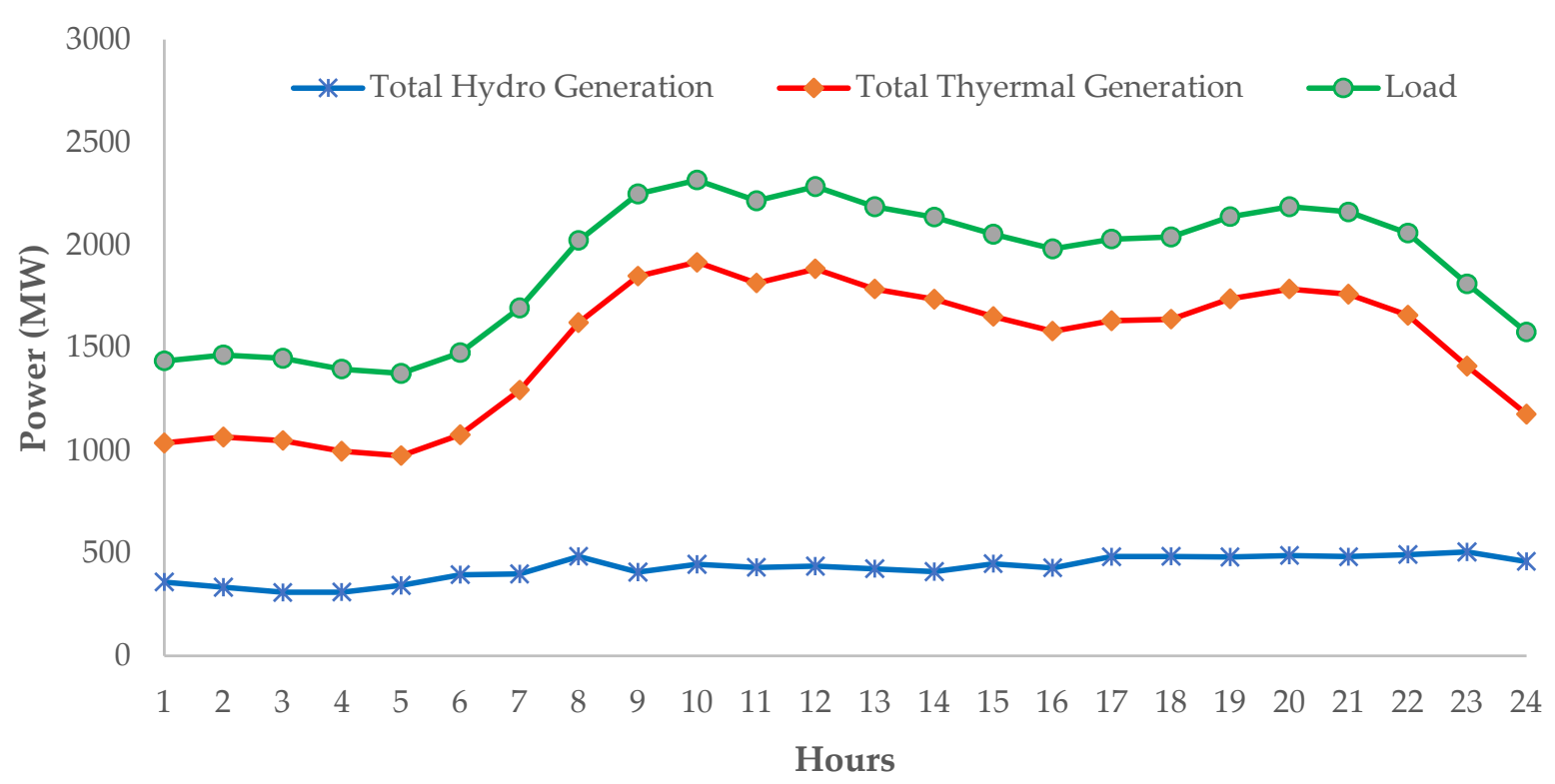

Figure 2. Hydro generation, thermal generation, and load demand for test system 1. 


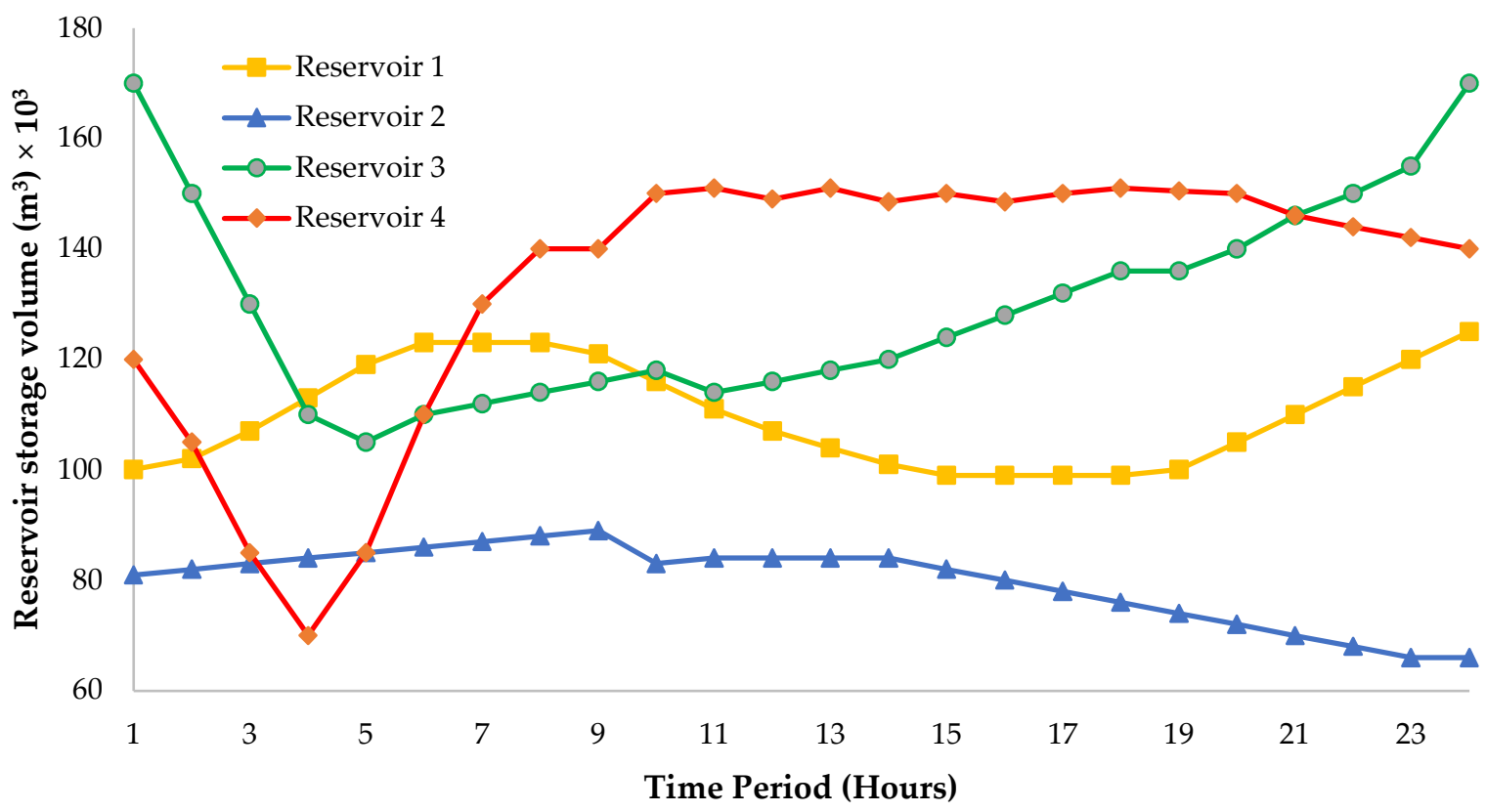

Figure 3. The reservoir storage volume of the hydro plants for test system 1.

\subsection{Proposed System Validation}

The obtained results of the proposed RCSA technique were compared with several other techniques (see Table 4). To maintain the correctness of this comparison, studies conducted for test system 1 were only considered. The gross cost obtained with RCSA was relatively equal to that produced by ORCSA-Lévy flights and ORCSA-Cauchy, and less than that produced by any other techniques. Nonetheless, the comparison results proved that the proposed RCSA technique is faster and more accurate in attaining solutions for StHS problems than the compared techniques.

Thus, the proposed technique is very efficient in making a solution for the StHS problem with reservoir volume constraints. Min/max cost is considered per day in USD, representing the best/worst fuel cost. The best/worst values are arranged by sorting from a more significant value to smallest depending on the minimum (best value) of fuel cost. Moreover, Table 4 represents the property of cost convergence for this suggested version of RCSA. The convergence comparison is shown that the RCSA technique was a more suitable optimal solution and more rapid than others.

In Table 5, the proposed RCSA technique's comparative results with other techniques for test system 2 were provided. Based on the results, it is understood that a decrease in cost is observed with RCSA. Complete results, involved to hourly water release, thermal and hydro generation, of the best solution achieved by the suggested RCSA please whole types of constraints of StHS problem while decreasing the gross fuel cost-efficiently. 
Table 4. Comparison of the results achieved by the suggested RCSA technique with others for test system 1.

\begin{tabular}{|c|c|c|c|c|}
\hline Techniques & Min Cost (\$) & Average Cost (\$) & Max Cost (\$) & CPU Time (s) \\
\hline GA [4] & 942,600 & $946,609.1$ & 951,087 & 1920 \\
\hline EGA [5] & $934,727.00$ & $936,058.00$ & $937,339.00$ & - \\
\hline FEP [6] & $930,267.92$ & $930,897.44$ & $931,396.81$ & 1911.2 \\
\hline CEP [6] & $930,166.25$ & $930,373.23$ & $930,927.01$ & 2292.1 \\
\hline IFEP [6] & $930,129.82$ & $930,290.13$ & $930,881.92$ & 1033.2 \\
\hline PSO [5] & $928,878.00$ & $933,085.00$ & $938,012.00$ & - \\
\hline CSA-Lévy [26] & $927,934.23$ & $927,980.45$ & $928,000.66$ & 79.08 \\
\hline CSA Cauchy [26] & $927,967.66$ & $927,981.49$ & $927,992.53$ & 81.30 \\
\hline CSA Gauss [26] & $927,957.26$ & $927,978.911$ & $928,003.23$ & 85.75 \\
\hline APSO [7] & $926,151.54$ & - & - & - \\
\hline EPSO [5] & $922,904.00$ & $923,527.00$ & $924,808.00$ & - \\
\hline MDE [8] & $922,555.44$ & - & - & - \\
\hline IPSO [9] & $922,553.49$ & - & - & - \\
\hline MAPSO [7] & $922,421.66$ & $922,544.00$ & $923,508.00$ & - \\
\hline TLBO [10] & $922,373.39$ & $922,462.24$ & $922,873.81$ & - \\
\hline CSA [20] & $913,945.87$ & $917,624.024$ & $921,994.25$ & - \\
\hline RIFEP [12] & $709,862.05$ & - & - & - \\
\hline GS [13] & $709,877.38$ & - & - & - \\
\hline SA [14] & $709,874.36$ & - & - & 901 \\
\hline $\begin{array}{l}\text { ORCSA-Lévy } \\
\text { flight [11] }\end{array}$ & $709,862.048$ & - & - & 18 \\
\hline $\begin{array}{l}\text { ORCSA-Cauchy } \\
\text { [11] }\end{array}$ & $709,862.048$ & - & - & 18 \\
\hline Proposed RCSA & $709,862.027$ & - & - & 17 \\
\hline
\end{tabular}

Table 5. Comparison of the results achieved by the suggested RCSA technique with others for test system 2 .

\begin{tabular}{lcccc}
\hline \multicolumn{1}{c}{ Techniques } & Min Cost (\$) & Average Cost (\$) & Max Cost (\$) & CPU Time (s) \\
\hline SA [27] & 47,306 & - & - & - \\
CEP [27] & 45,466 & - & - & - \\
CEP-IFS [25] & $45,036.00$ & - & - & - \\
PSO [27] & 44,740 & - & - & - \\
DE [28] & $44,526.1$ & - & - & 200 \\
MDE [28] & $42,611.14$ & - & - & 125 \\
CSR [15] & 42,440 & - & - & 109 \\
TLBO [10] & $42,385.88$ & $42,407.23$ & $42,441.36$ & - \\
HDE [28] & $42,337.3$ & - & - & 48 \\
SQP [17] & $42,120.02$ & - & - & 625.07 \\
KHA [16] & 41,926 & - & - & - \\
MHDE [28] & $41,856.5$ & - & - & 31 \\
CSA [25] & $41,046.897$ & - & - & 94.4 \\
Proposed RCSA & $41,013.09$ & $41,401.5$ & $41,789.9$ & 17 \\
\hline
\end{tabular}

Besides the above comparison made in Tables 4 and 5 for the test systems 1 and 2, we provided a comparative discussion with recent works available in the literature. Firstly, it is challenging to sketch them in a single unique figure because there are many output costs with various $\mathrm{X}-\mathrm{Y}$ axis points. Thus, we provided the most suitable algorithms that are in comparison with the proposed one. H.M Dubey et al. [20] applied conventional CSA. It was found that the cost convergence characteristic is $\$ 2.5 \times 10^{7}$, which is then starting to reduce until it gets to $\$ 2.5 \times 10^{7}$. Additionally, the conventional CSA was employed in the same system but with various parameters and different specialized system constraints and fitness function (for example, the fixed-head). Here, the cost convergence characteristic was restricted from $\$ 2 \times 10^{11}$ to $0.1 \times 10^{11}$ [27]. T.T Nguyen used the modified CSA and D. 
$\mathrm{N}$ Vo in ref [29]; it was observed that the fitness function's cost convergence started from $\$ 3.9 \times 10^{5}$ and got settled at $\$ 3.7 \times 10^{5}$.

Finally, Figure 4 describes the cost convergence characteristic for some contribution that has the same range of our proposed algorithm and this was done mainly to see the validation. Then again, T.T Nguyen has applied the same with one rank on CSA [11]; and an adaptive selective approach on CSA [30].

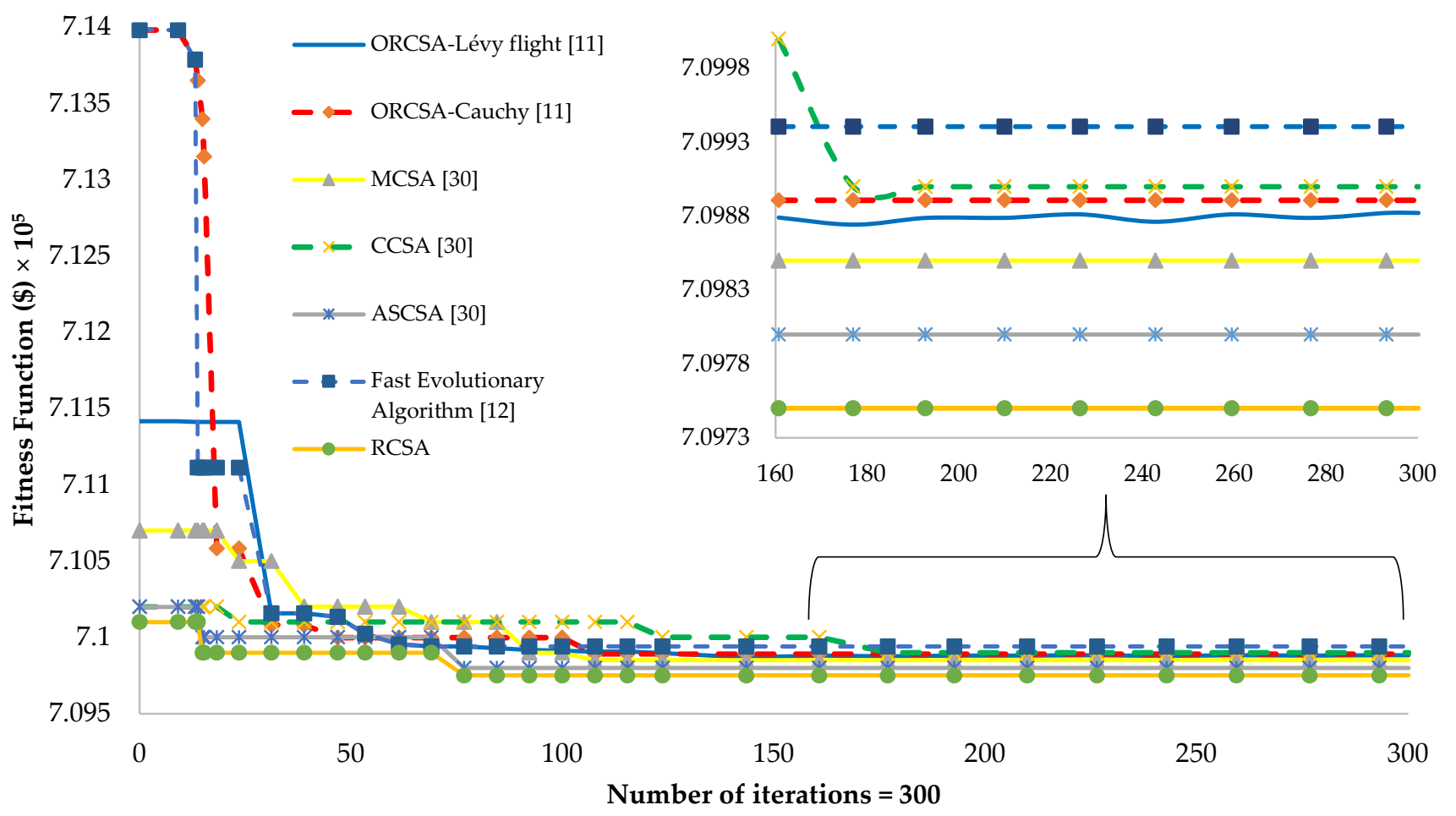

Figure 4. Convergence characteristic of the five various algorithms with RCSA methods.

\section{Conclusions}

In this study, the RCSA technique was implemented to solve the StHS problems with various complex constraints. The same was tested with four cascaded hydropower plants and one thermal plant for the scheduled operation of $24 \mathrm{~h}$ with subintervals of $1 \mathrm{~h}$. The results proved that the proposed RCSA technique was more effective than the conventional CSA for the StHS problem. Simulation results of the cascaded hydrothermal systems have shown that the RCSA technique achieved a satisfactory optimal solution where the computing time was lesser than other recent optimization techniques.

Author Contributions: Conceptualization, A.T.H., A.N.K., and N.M.K.; methodology, C.Z., A.T.H., and, N.M.K.; software, C.Z., and M.J.; validation, A.T.H., and N.M.K.; formal analysis, A.T.H., A.N.K., and N.M.K.; investigation, C.Z., A.T.H., A.N.K., and M.N.M.; data curation, A.T.H.; writing —original draft preparation, A.T.H., C.Z., and N.M.K.; writing-review and editing, N.M.K., A.N.K., M.J., and M.N.M.; visualization, N.M.K.; supervision, N.M.K.; project administration, N.M.K., M.J., M.N.M., funding acquisition, C.Z., M.J., M.N.M., and N.M.K. All authors have read and agreed to the published version of the manuscript.

Funding: This work was sponsored by Qing Lan Project of JiangSu Province (No.20200420), Opening Project of Henan Engineering Laboratory of Photoelectric Sensor and Intelligent Measurement and Control of Henan Polytechnic University (No.HELPSIMC-2020-002).

Institutional Review Board Statement: Not applicable.

Informed Consent Statement: Not applicable.

Data Availability Statement: Data sharing not applicable. 
Conflicts of Interest: The authors declare no conflict of interest.

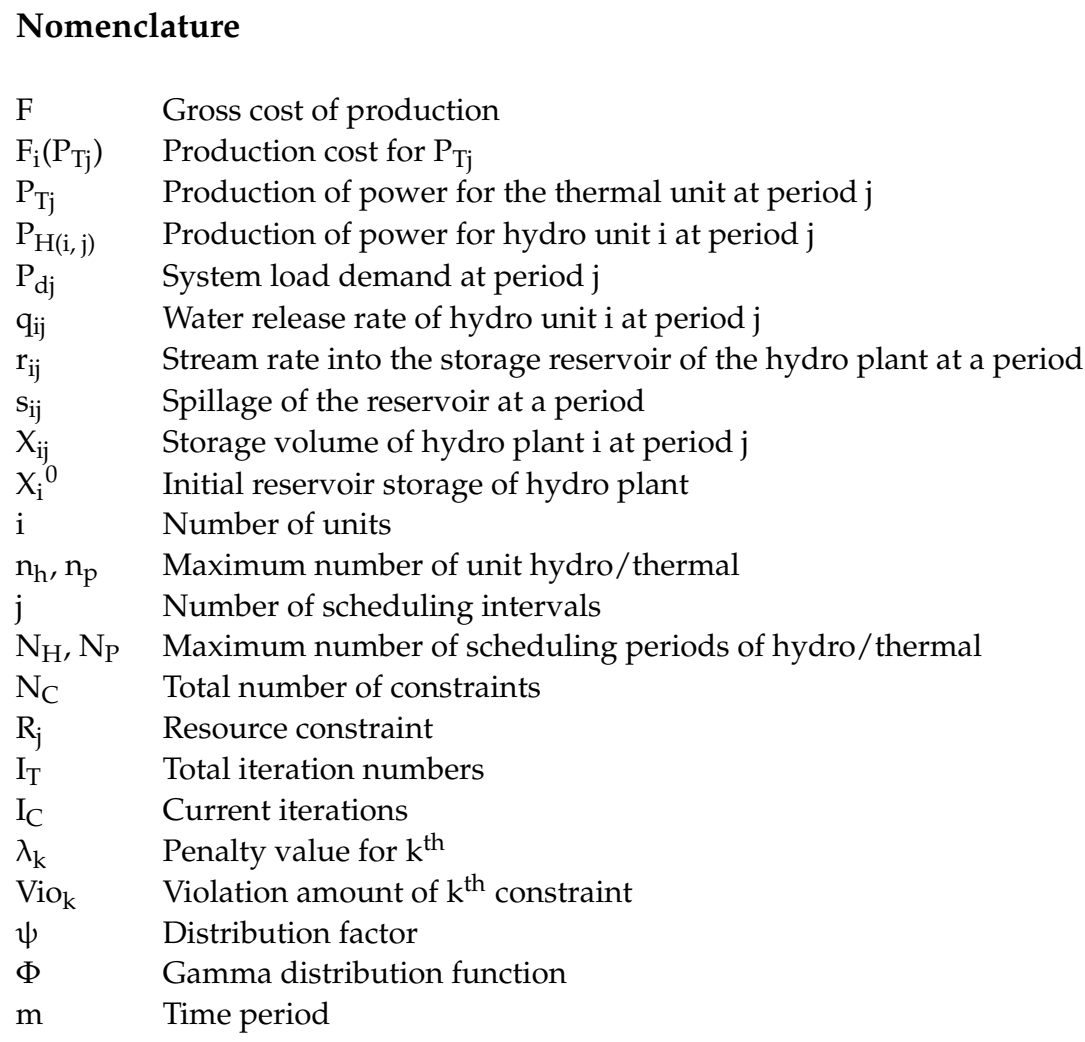

\section{References}

1. Hammid, A.T.; Bin Sulaiman, M.H.; Abdalla, A.N. Prediction of small hydropower plant power production in Himreen Lake dam (HLD) using artificial neural network. Alex. Eng. J. 2018, 57, 211-221. [CrossRef]

2. Hammid, A.T.; Kadhim, M.B.H.; Sulaiman, A.A.; Kareem, A.N.; Jadaa, K.J. Priority of kaplan turbine and small hydropower plants over other resources: An overview. J. Eng. Appl. Sci. 2017, 12, 8695-8705.

3. Thaeer Hammid, A.; Awad, O.I.; Sulaiman, M.H.; Gunasekaran, S.S.; Mostafa, S.A.; Manoj Kumar, N.; Khalaf, B.A.; Al-Jawhar, Y.A.; Abdulhasan, R.A. A review of optimization algorithms in solving hydro generation scheduling problems. Energies 2020, 13, 2787. [CrossRef]

4. Orero, S.; Irving, M. A genetic algorithm modelling framework and solution technique for short term optimal hydrothermal scheduling. IEEE Trans. Power Syst. 1998, 13, 501-518. [CrossRef]

5. Yuan, X.; Wang, L.; Yuan, Y. Application of enhanced PSO approach to optimal scheduling of hydro system. Energy Convers. Manag. 2008, 49, 2966-2972. [CrossRef]

6. Sinha, N.; Chakrabarti, R.; Chattopadhyay, P. Fast evolutionary programming techniques for short-term hydrothermal scheduling. IEEE Trans. Power Syst. 2003, 18, 214-220. [CrossRef]

7. Amjady, N.; Soleymanpour, H.R. Daily hydrothermal generation scheduling by a new modified adaptive particle swarm optimization technique. Electr. Power Syst. Res. 2010, 80, 723-732. [CrossRef]

8. Lakshminarasimman, L.; Subramanian, S. Short-term scheduling of hydrothermal power system with cascaded reservoirs by using modified differential evolution. IEE Proc. Gener. Transm. Distrib. 2006, 153, 693-700. [CrossRef]

9. Hota, P.; Barisal, A.; Chakrabarti, R. An improved PSO technique for short-term optimal hydrothermal scheduling. Electr. Power Syst. Res. 2009, 79, 1047-1053. [CrossRef]

10. Roy, P.K. Teaching learning based optimization for short-term hydrothermal scheduling problem considering valve point effect and prohibited discharge constraint. Int. J. Electr. Power Energy Syst. 2013, 53, 10-19. [CrossRef]

11. Nguyen, T.T.; Vo, D.N.; Ongsakul, W.; Trung, N.T. One rank cuckoo search algorithm for short-term hydrothermal scheduling with reservoir constraint. In Proceedings of the IEEE Eindhoven PowerTech, Eindhoven, The Netherlands, 29 June-2 July 2015.

12. Türkay, B.; Mecitoğlu, F.; Baran, S. Application of a fast evolutionary algorithm to short-term hydro-thermal generation scheduling Energy Sources Part B Econ. Planning, Policy 2011, 6, 395-405. [CrossRef]

13. Wood, A.J.; Wollenberg, B.F.; Sheblé, G.B. Power Generation, Operation, and Control; John Wiley \& Sons: Hoboken, NJ, USA, 2013.

14. Wong, K.; Wong, Y. Short-term hydrothermal scheduling part. I. Simulated annealing approach. IEE Proc. Gener. Transm. Distrib. 1994, 141, 497-501. [CrossRef] 
15. Swain, R.; Barisal, A.; Hota, P.; Chakrabarti, R. Short-term hydrothermal scheduling using clonal selection algorithm. Int. J. Electr. Power Energy Syst. 2011, 33, 647-656. [CrossRef]

16. Das, S.; Bhattacharya, A. Symbiotic organisms search algorithm for short-term hydrothermal scheduling. Ain Shams Eng. J. 2018, 9, 499-516. [CrossRef]

17. Sivasubramani, S.; Swarup, K.S. Hybrid DE-SQP algorithm for non-convex short term hydrothermal scheduling problem. Energy Convers. Manag. 2011, 52, 757-761. [CrossRef]

18. Yang, X.-S.; Deb, S. Cuckoo search via Lévy flights. In Proceedings of the 2009 World Congress on Nature \& Biologically Inspired Computing (NaBIC), Coimbatore, India, 9-11 December 2009.

19. Vo, D.N.; Schegner, P.; Ongsakul, W. Cuckoo search algorithm for non-convex economic dispatch. IET Gener. Transm. Distrib. 2013, 7, 645-654. [CrossRef]

20. Dubey, H.M.; Pandit, M.; Panigrahi, B. Cuckoo Search Algorithm for Short Term Hydrothermal Scheduling. In Power Electronics and Renewable Energy Systems; Springer: Berlin/Heidelberg, Germany, 2015; pp. 573-589.

21. Xinchun, L.; Yongde, K.; Hongna, C.; Hui, L. Hydrothermal Effects of Freeze-Thaw in the Taklimakan Desert. Sustainability 2021, 13, 1292. [CrossRef]

22. Zeng, X.; Hammid, A.T.; Kumar, N.M.; Subramaniam, U.; Almakhles, D.J. A grasshopper optimization algorithm for optimal short-term hydrothermal scheduling. Energy Rep. 2021, 7, 314-323. [CrossRef]

23. Zhang, Z.; Fan, W.; Bao, W.; Chen, C.-T.; Liu, S.; Cai, Y. Recent Developments of Exploration and Detection of Shallow-Water Hydrothermal Systems. Sustainability 2020, 12, 9109. [CrossRef]

24. Kanazawa, K.; Sano, T.G.; Cairoli, A.; Baule, A. Loopy Lévy flights enhance tracer diffusion in active suspensions. Nature 2020, 579, 364-367. [CrossRef]

25. Nguyen, T.T.; Vo, D.N.; Dinh, B.H. A cuckoo bird-inspired meta-heuristic algorithm for optimal short-term hydrothermal generation cooperation. Cogent Eng. 2016, 3, 1266863. [CrossRef]

26. Nguyen, T.T.; Vo, D.N.; Dao, T.T.; Dieu, V.N. Cuckoo search algorithm using different distributions for short-term hydrothermal scheduling with cascaded hydropower plants. In Proceedings of the TENCON 2014-2014 IEEE Region 10 Conference, Bangkok, Thailand, 22-25 October 2014.

27. Nguyen, T.T.; Vo, D.N.; Truong, A.V. Cuckoo search algorithm for short-term hydrothermal scheduling. Appl. Energy 2014, 132, 276-287. [CrossRef]

28. Basu, M. Economic environmental dispatch of hydrothermal power system. Int. J. Electr. Power Energy Syst. 2010, 32, 711-720. [CrossRef]

29. Nguyen, T.T.; Vo, D.N. Modified cuckoo search algorithm for short-term hydrothermal scheduling. Int. J. Electr. Power Energy Syst. 2015, 65, 271-281. [CrossRef]

30. Nguyen, T.T.; Vo, D.N.; Dinh, B.H. An effectively adaptive selective cuckoo search algorithm for solving three complicated short-term hydrothermal scheduling problems. Energy 2018, 155, 930-956. [CrossRef] 